\title{
Bromine Substituent Position Triggered Halogen versus Hydrogen Bond in 2D Self-Assembly of Fluorenone Derivatives
}

\author{
Meiqiu Dong, Juntian Wu, Xinrui Miao, * Jinxing Li, Wenli Deng*
}

College of Materials Science and Engineering, South China University of Technology, Guangzhou 510640, People's Republic of China

*E-mail: msxrmiao@scut.edu.cn

*E-mail: wldeng@scut.edu.cn 


\section{1. ${ }^{1} \mathrm{H}$ NMR data of $\mathrm{X}-\mathrm{BFC15}(\mathrm{X}=1,2$, and 3)}

\section{1-BFC15}

${ }^{1} \mathrm{H}$ NMR (600 MHz, $\left.\mathrm{CDCl}_{3}, \delta\right): 7.38(\mathrm{~d}, \mathrm{~J}=8.2 \mathrm{~Hz}, 1 \mathrm{H} ; \mathrm{Ar} \mathrm{H}), 7.34(\mathrm{dd}, \mathrm{J}=7.3,0.8 \mathrm{~Hz}, 1 \mathrm{H} ; \mathrm{Ar} \mathrm{H})$, $7.29(\mathrm{dd}, \mathrm{J}=8.0,0.8 \mathrm{~Hz}, 1 \mathrm{H} ; \mathrm{Ar} H), 7.23(\mathrm{dd}, \mathrm{J}=8.0,7.3 \mathrm{~Hz}, 1 \mathrm{H} ; \mathrm{Ar} H), 7.18(\mathrm{~d}, \mathrm{~J}=2.3 \mathrm{~Hz}, 1 \mathrm{H} ; \mathrm{Ar}$ H), $6.98(\mathrm{dd}, \mathrm{J}=8.2,2.5 \mathrm{~Hz}, 1 \mathrm{H} ; \mathrm{Ar} \mathrm{H}), 3.99\left(\mathrm{t}, \mathrm{J}=6.5 \mathrm{~Hz}, 2 \mathrm{H} ; \mathrm{CH}_{2}\right), 1.75-1.79\left(\mathrm{~m}, 2 \mathrm{H} ; \mathrm{CH}_{2}\right)$, $1.41-1.46\left(\mathrm{~m}, 2 \mathrm{H} ; \mathrm{CH}_{2}\right), 1.31-1.35\left(\mathrm{~m}, 2 \mathrm{H} ; \mathrm{CH}_{2}\right), 1.24-1.30\left(\mathrm{~m}, 20 \mathrm{H} ; \mathrm{CH}_{2}\right), 0.84-0.87(\mathrm{t}, \mathrm{J}=7.0 \mathrm{~Hz}$, $\left.3 \mathrm{H} ; \mathrm{CH}_{3}\right)$.

\section{2-BFC15}

${ }^{1} \mathrm{H}$ NMR (600 MHz, $\left.\mathrm{CDCl}_{3}, \delta\right): 7.67$ (d, J=1.8 Hz, 1H; Ar H), 7.53 (dd, J = 7.9, 1.9 Hz, 1H; Ar H), $7.36(\mathrm{~d}, \mathrm{~J}=8.2 \mathrm{~Hz}, 1 \mathrm{H} ; \operatorname{Ar} \mathrm{H}), 7.25(\mathrm{~d}, \mathrm{~J}=7.8 \mathrm{~Hz}, 1 \mathrm{H} ; \mathrm{Ar} \mathrm{H}), 7.16(\mathrm{~d}, \mathrm{~J}=2.4 \mathrm{~Hz}, 1 \mathrm{H} ; \mathrm{Ar} \mathrm{H}), 6.97$ (dd, $\mathrm{J}=8.2,2.5 \mathrm{~Hz}, 1 \mathrm{H} ; \mathrm{Ar} \mathrm{H}), 3.98\left(\mathrm{t}, \mathrm{J}=6.6 \mathrm{~Hz}, 2 \mathrm{H} ; \mathrm{CH}_{2}\right), 1.74-1.79\left(\mathrm{~m}, 2 \mathrm{H} ; \mathrm{CH}_{2}\right), 1.40-1.45(\mathrm{~m}, 2 \mathrm{H}$; $\left.\mathrm{CH}_{2}\right), 1.31-1.34\left(\mathrm{~m}, 2 \mathrm{H} ; \mathrm{CH}_{2}\right), 1.24-1.30\left(\mathrm{~m}, 20 \mathrm{H} ; \mathrm{CH}_{2}\right), 0.85-0.87\left(\mathrm{t}, \mathrm{J}=7.0 \mathrm{~Hz}, 3 \mathrm{H} ; \mathrm{CH}_{3}\right)$.

\section{3-BFC15}

${ }^{1} \mathrm{H}$ NMR (600 MHz, $\mathrm{CDCl}_{3}, \delta$ ): 7.52 (d, J = 1.5 Hz, 1H; Ar H), 7.43 (d, J = 7.8 Hz, 1H; Ar H), 7.35 $(\mathrm{d}, \mathrm{J}=8.2 \mathrm{~Hz}, 1 \mathrm{H} ;$ Ar H), $7.32(\mathrm{dd}, \mathrm{J}=7.8,1.6 \mathrm{~Hz}, 1 \mathrm{H} ; \operatorname{Ar} \mathrm{H}), 7.17(\mathrm{~d}, \mathrm{~J}=2.4 \mathrm{~Hz}, 1 \mathrm{H} ; \operatorname{Ar} \mathrm{H}), 6.97$ $(\mathrm{dd}, \mathrm{J}=8.2,2.5 \mathrm{~Hz}, 1 \mathrm{H} ; \mathrm{Ar} \mathrm{H}), 3.99\left(\mathrm{t}, \mathrm{J}=6.6 \mathrm{~Hz}, 2 \mathrm{H} ; \mathrm{CH}_{2}\right), 1.74-1.79\left(\mathrm{~m}, 2 \mathrm{H} ; \mathrm{CH}_{2}\right), 1.40-1.45$ (m, $\left.2 \mathrm{H} ; \mathrm{CH}_{2}\right), 1.31-1.34\left(\mathrm{~m}, 2 \mathrm{H} ; \mathrm{CH}_{2}\right), 1.24-1.30\left(\mathrm{~m}, 20 \mathrm{H} ; \mathrm{CH}_{2}\right), 0.84-0.87\left(\mathrm{t}, \mathrm{J}=7.2 \mathrm{~Hz}, 3 \mathrm{H} ; \mathrm{CH}_{3}\right)$. 


\section{Additional STM images}

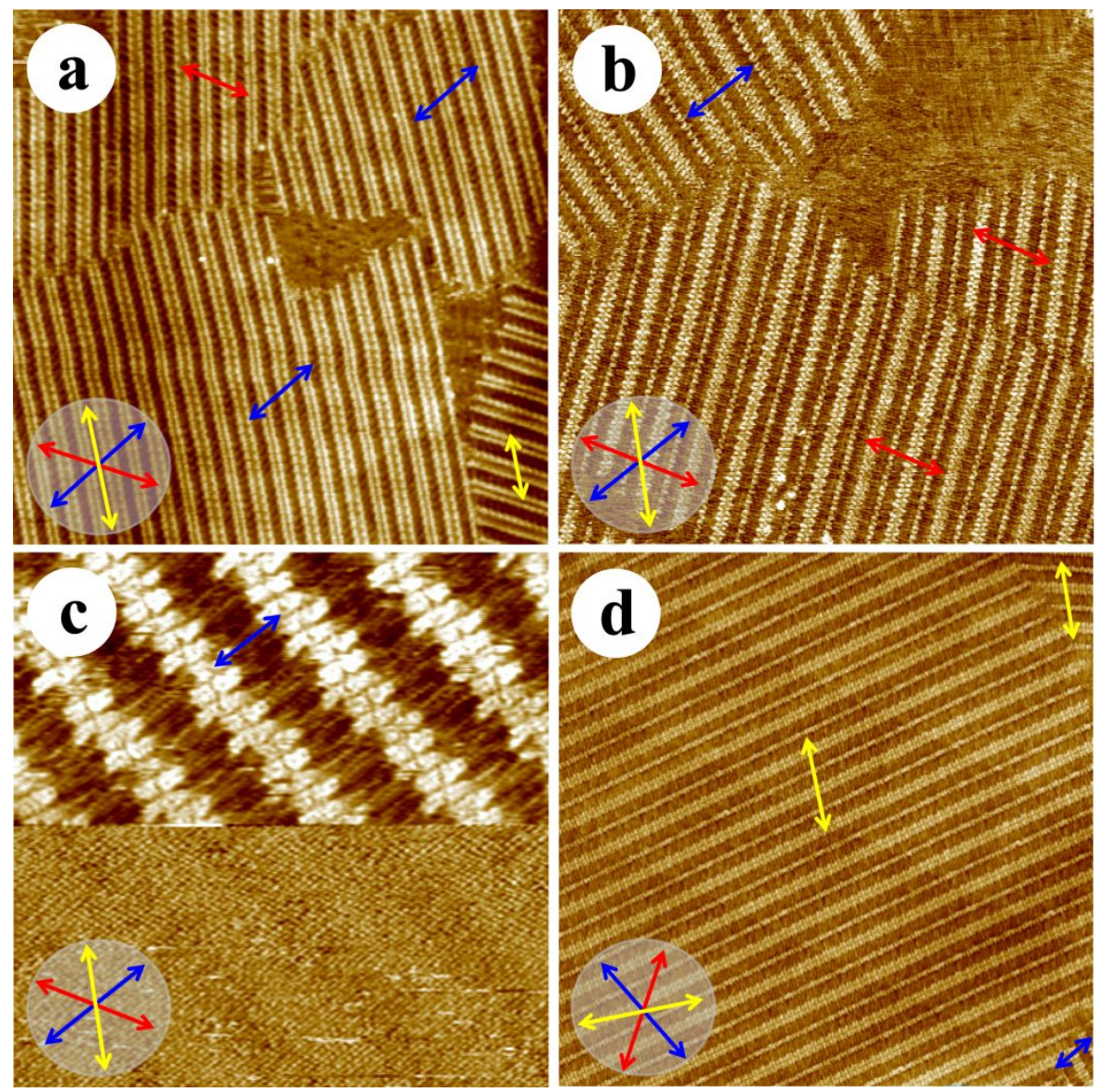

Figure S1. (a) Large-scale STM image of the alternate-I pattern for 1-BFC15. Arrows show the orientation of alkoxy chains in the stripes at both sides of the two-row troughs. $\left(2.6 \times 10^{-4} \mathrm{M} ; I_{\mathrm{t}}=493\right.$ $\mathrm{pA}, V_{\mathrm{b}}=605 \mathrm{mV} ; 100 \times 100 \mathrm{~nm}^{2}$ ). (b) Large-scale STM image of the alternate-II pattern for 2-BFC15. $\left(5.1 \times 10^{-4} \mathrm{M} ; I_{\mathrm{t}}=511 \mathrm{pA}, V_{\mathrm{b}}=600 \mathrm{mV} ; 100 \times 100 \mathrm{~nm}^{2}\right) .(\mathrm{c})$ High-resolution STM image of the coexistence of the lamellar pattern for 3-BFC15 and HOPG surface. $\left(6.1 \times 10^{-4} \mathrm{M} ; I_{\mathrm{t}}=451 \mathrm{pA}, V_{\mathrm{b}}=\right.$ $\left.625 \mathrm{mV} ; 20 \times 20 \mathrm{~nm}^{2}\right)$. (d) Large-scale STM image of the alternate-III pattern for 3-BFC15. $(6.1 \times$ $\left.10^{-4} \mathrm{M} ; I_{\mathrm{t}}=451 \mathrm{pA}, V_{\mathrm{b}}=625 \mathrm{mV} ; 100 \times 100 \mathrm{~nm}^{2}\right)$. Arrows in $(\mathrm{b}, \mathrm{c}, \mathrm{d})$ show the orientation of corresponding alkoxy chains. Arrows with similar color in each image display the similar orientation. Bottom-left insets represente the HOPG lattices. 


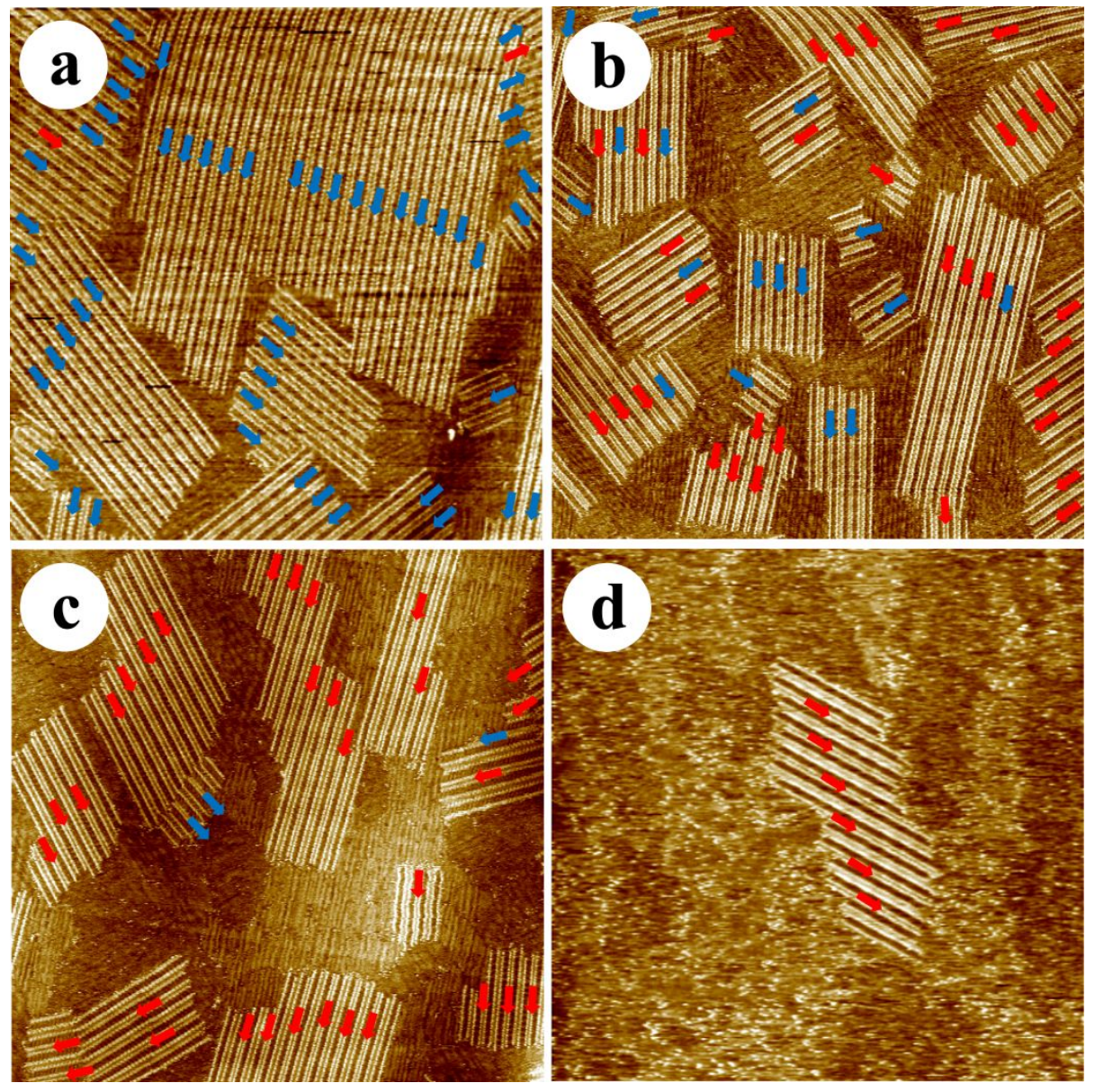

Figure S2. Large-scale STM images of 1-BFC15 self-assembled into the alternate-I pattern at the 1octanoic acid/HOPG interface. Scale bar: $200 \times 200 \mathrm{~nm}^{2}$. (a) Concentration: $2.6 \times 10^{-4} \mathrm{M}$; Tunneling parameters: $I_{\mathrm{t}}=493 \mathrm{pA}, V_{\mathrm{b}}=605 \mathrm{mV}$; (b) $8.4 \times 10^{-5} \mathrm{M}$; $I_{\mathrm{t}}=502 \mathrm{pA}, V_{\mathrm{b}}=622 \mathrm{mV}$; (c) $1.9 \times 10^{-5} \mathrm{M}$; $I_{\mathrm{t}}=488 \mathrm{pA}, V_{\mathrm{b}}=636 \mathrm{mV}$; (d) $3.7 \times 10^{-6} \mathrm{M} ; I_{\mathrm{t}}=475 \mathrm{pA}, V_{\mathrm{b}}=624 \mathrm{mV}$. The looser two-row troughs are indicated by the red arrows, while the denser two-row troughs are marked with the blue arrows. 


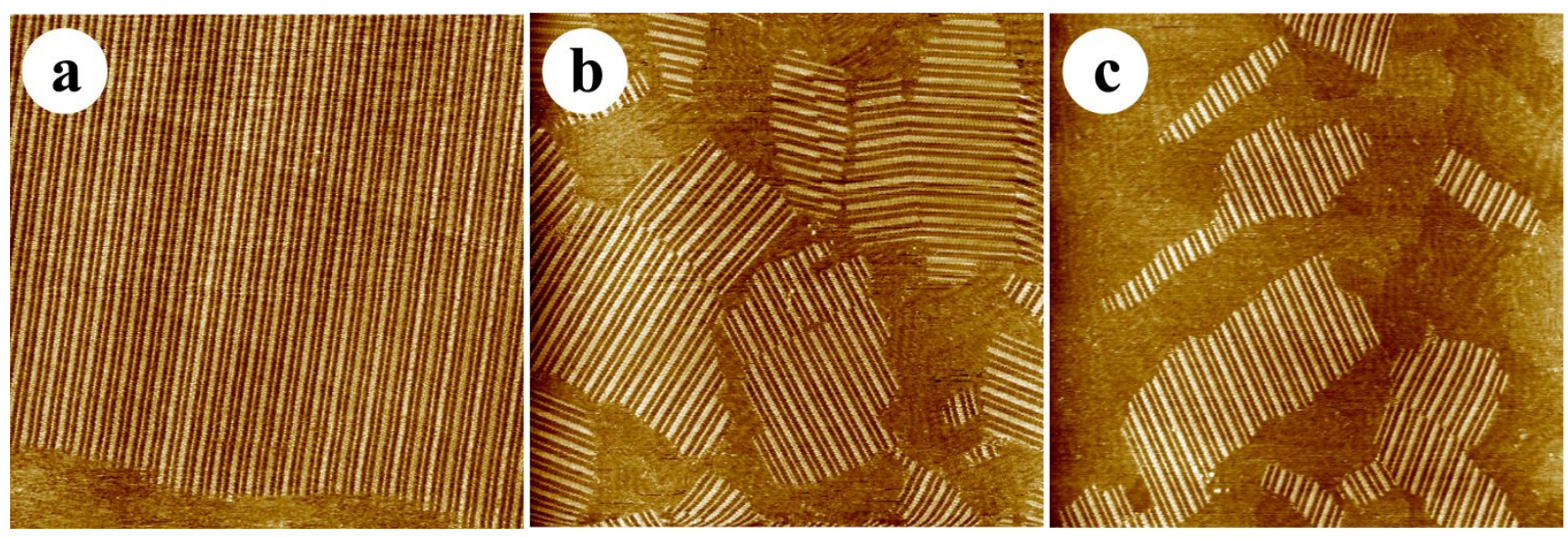

Figure S3. Large-scale STM images of 2-BFC15 self-assembled into the alternate-II pattern at the 1octanoic acid/HOPG interface. Scale bar: $200 \times 200 \mathrm{~nm}^{2}$. (a) $5.1 \times 10^{-4} \mathrm{M} ; I_{\mathrm{t}}=511 \mathrm{pA}, V_{\mathrm{b}}=600 \mathrm{mV}$; (b) $2.6 \times 10^{-5} \mathrm{M} ; I_{\mathrm{t}}=539 \mathrm{pA}, V_{\mathrm{b}}=640 \mathrm{mV}$; (c) $7.9 \times 10^{-6} \mathrm{M} ; I_{\mathrm{t}}=497 \mathrm{pA}, V_{\mathrm{b}}=635 \mathrm{mV}$.
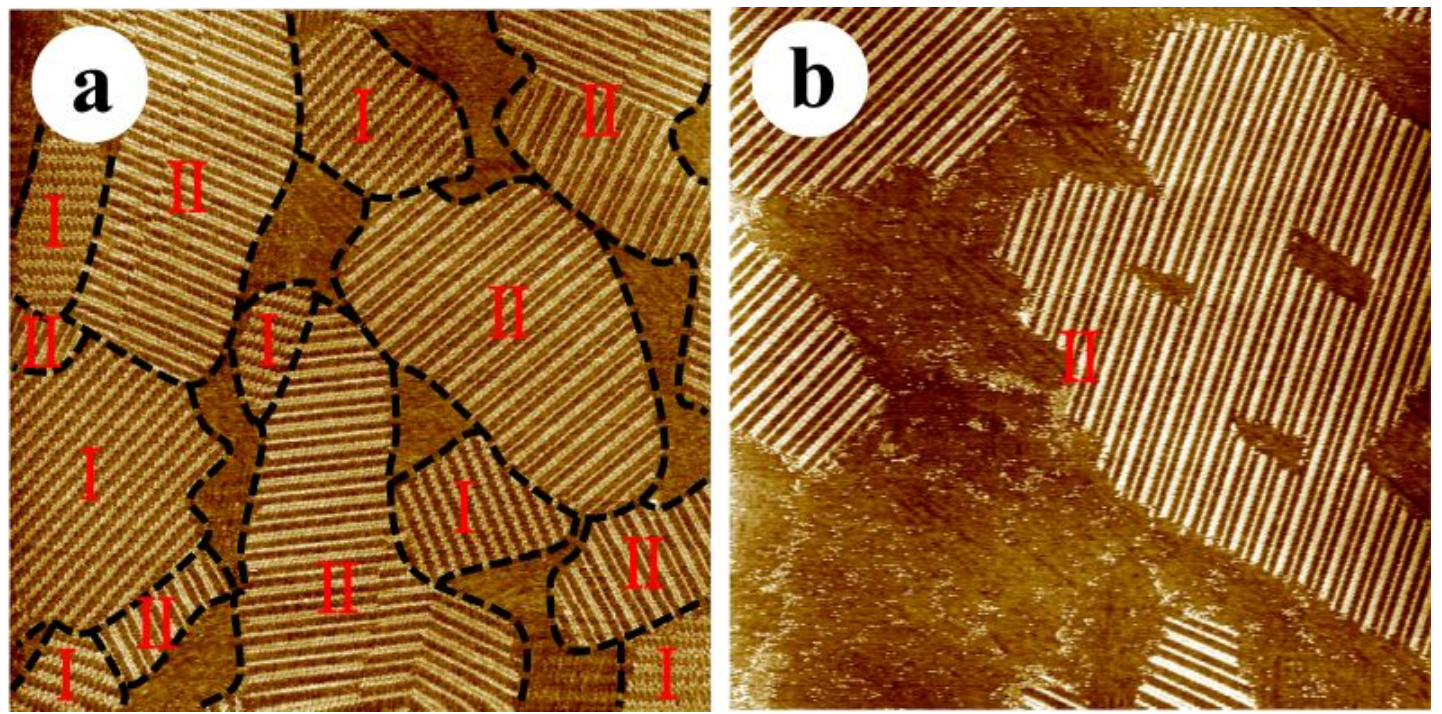

Figure S4. (a) Coexisted well-defined self-assembly structures of the lamellar pattern (labeled I) and the alternate-III pattern (labeled II) for 3-BFC15 at the 1 -octanoic acid/HOPG interface $\left(6.1 \times 10^{-4} \mathrm{M}\right.$; $I_{\mathrm{t}}=451 \mathrm{pA}, V_{\mathrm{b}}=625 \mathrm{mV} ; 200 \times 200 \mathrm{~nm}^{2}$ ). (b) Large-scale STM image of the alternate-III pattern for 3 -BFC15 at the 1-octanoic acid/HOPG interface $\left(2.5 \times 10^{-5} \mathrm{M} ; I_{\mathrm{t}}=433 \mathrm{pA}, V_{\mathrm{b}}=601 \mathrm{mV} ; 200 \times 200\right.$ $\left.\mathrm{nm}^{2}\right)$. 